International Journal of Modern Physics D

(C) World Scientific Publishing Company

\title{
CAN A VARIABLE GRAVITATIONAL CONSTANT RESOLVE THE FAINT YOUNG SUN PARADOX ?*
}

\author{
VARUN SAHNI \\ Inter-University Centre for Astronomy and Astrophysics, \\ Post Bag 4, Ganeshkhind, Pune 411 007, India \\ varun@iucaa.ernet.in \\ YURI SHTANOV ${ }^{\dagger}$ \\ Bogolyubov Institute for Theoretical Physics, \\ 14-b Metrologicheskaya St., Kiev 03680, Ukraine \\ Department of Physics, Taras Shevchenko National University of Kiev, \\ 64/13 Vladimirskaya St., Kiev 01601, Ukraine \\ shtanov@bitp.kiev.ua
}

Received Day Month Year

Revised Day Month Year

\begin{abstract}
Solar models suggest that four billion years ago the young Sun was $\sim 25 \%$ fainter than it is today, rendering Earth's oceans frozen and lifeless. However, there is ample geophysical evidence that Earth had a liquid ocean teeming with life 4 Gyr ago. Since $\mathcal{L}_{\odot} \propto G^{7} M_{\odot}^{5}$, the Sun's luminosity $\mathcal{L}_{\odot}$ is exceedingly sensitive to small changes in the gravitational constant $G$. We show that a percent-level increase in $G$ in the past would have prevented Earth's oceans from freezing, resolving the faint young Sun paradox. Such small changes in $G$ are consistent with observational bounds on $\Delta G / G$. Since $\mathcal{L}_{\mathrm{SNIa}} \propto G^{-3 / 2}$, an increase in $G$ leads to fainter supernovae, creating tension between standard candle and standard ruler probes of dark energy. Precisely such a tension has recently been reported by the Planck team.
\end{abstract}

Keywords: Faint Young Sun Paradox; variable gravity; dark energy.

PACS numbers: 92.60.Iv, 92.70.Qr, 95.30.Sf, 96.60.Q-

\section{The Faint Young Sun Paradox}

Between 4.5 and 3.9 billion years ago was an exciting time in the history of our planet. This period witnessed the assembly of the Earth from planetesimals, the creation of the moon, the formation of Earth's primeval oceans, etc. This was also when the Sun became a main-sequence star.

*This essay received an honorable mention in the 2014 Essay Competition of the Gravity Research Foundation.

${ }^{\dagger}$ Corresponding author. 
The early Earth, however, faced a severe problem - that of the faint young Sun ${ }^{[12]}$ Numerical models of the Sun's interior suggest that solar luminosity about 4 Gyr ago (the Archean epoch), was only about $75 \%$ of its present value. This would have affected our oceans dramatically, reducing their temperature to $268^{\circ} \mathrm{K}$ and ensuring that they were completely frozen during much of the Archean. ${ }^{3}$

However, geophysical and climatological data do not support this view. On the contrary, all evidence points to a temperate climate on Earth with liquid oceans teeming with primordial life forms $\sim 4$ Gyr ago. Thus, the presence of metamorphosed sedimentary rocks (from the Archean) shows strong evidence of erosion by liquid water. More evidence comes from fossils of early life forms which depended upon water to survive! 1

Evidence for a temperate climate on Earth also comes from the abundance of stable isotopes of Oxygen, ${ }^{16} \mathrm{O},{ }^{17} \mathrm{O}$ and ${ }^{18} \mathrm{O}$. Water on our planet is a mixture of $\mathrm{H}_{2}{ }^{16} \mathrm{O}, \mathrm{H}_{2}{ }^{17} \mathrm{O}$ and $\mathrm{H}_{2}{ }^{18} \mathrm{O}$, with the former being the most abundant. However, $\mathrm{H}_{2}{ }^{16} \mathrm{O}$ is also preferentially evaporated from our oceans which should, consequently, have been enriched in ${ }^{18} \mathrm{O}$ during the prolonged ice ages which accompanied a fainter Sun. The ${ }^{16} \mathrm{O} /{ }^{18} \mathrm{O}$ ratio, therefore, records Earth's temperature through prehistory.

Luckily, relics of tiny sea creatures "coccolithophorids" can preserve the primordial ${ }^{16} \mathrm{O} /{ }^{18} \mathrm{O}$ ratio through their calcite $\left(\mathrm{CaCO}_{3}\right)$ shells, which absorb ${ }^{16} \mathrm{O}$ and ${ }^{18} \mathrm{O}$

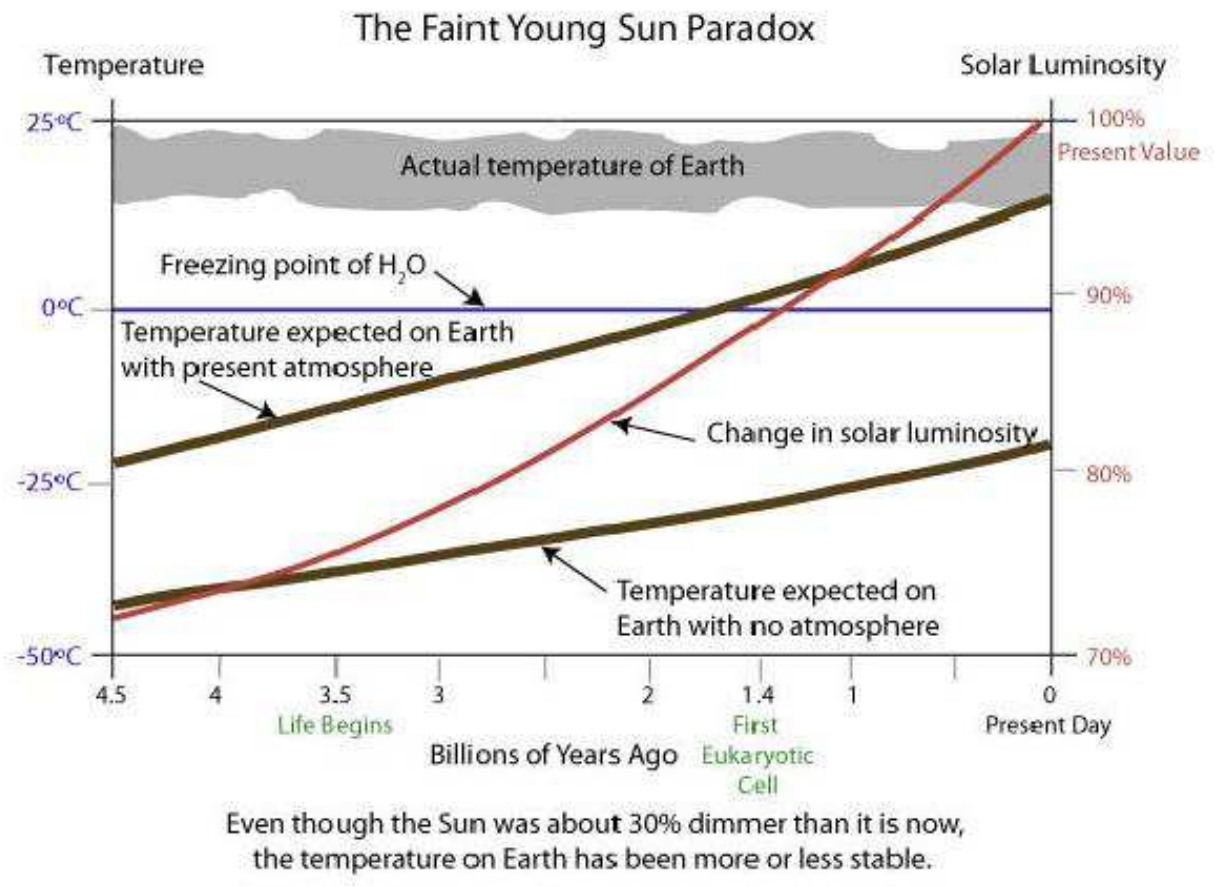

Fig. 1. Illustration of the Faint Young Sun Paradox. 
in equal amounts. Similarly, cherts, rocks composed of silica, also bear testimony to the ${ }^{16} \mathrm{O} /{ }^{18} \mathrm{O}$ ratio in the water in which these rocks precipitated. Both the chert and $\mathrm{CaCO}_{3}$ data imply that the Earth's temperature has remained roughly unchanged over the past 4 Gyr, which challenges the faint young Sun hypothesis, $\stackrel{4}{[}$ see Fig. 1.

\section{A Greenhouse Effect on Early Earth ?}

While several arguments have been advanced to resolve the Faint Young Sun Paradox, none is problem-free. For instance, one might try to compensate for the diminished sunlight by assuming that Earth's early atmosphere had a far greater concentration of greenhouse gases, such as $\mathrm{CO}_{2}$, than at present.

However this hypothesis runs into difficulties. In order to maintain the Earth's temperature above the freezing point of water during the early Archean, the abundance of $\mathrm{CO}_{2}$ in Earth's primeval atmosphere should have been a thousand times its present value. One must, therefore, account for the enormous decline in the $\mathrm{CO}_{2}$ concentration: from roughly 10 atmospheres 4 Gyr ago, to $3 \times 10^{-3}$ atmospheres at present.

While $\mathrm{CO}_{2}$ can be removed from the atmosphere by bacteria and plants during photosynthesis and also by the weathering of rock, the enormous concentrations referred to earlier seem difficult to account for. Indeed, large amounts of $\mathrm{CO}_{2}$ in the early atmosphere would have led to the formation of the iron carbonate based compound siderite $\left(\mathrm{FeCO}_{3}\right)$. However, analysis of billion year old paleosols challenges this picture by finding no $\mathrm{FeCO}_{3}$. Found instead are iron silicates which support a much more moderate presence of $\mathrm{CO}_{2}$ in the early atmosphere. Moreover, it would be difficult to make $\mathrm{CO}_{2}$ disappear almost entirely from the Earth's atmosphere since much of it, after reacting with rocks and being used by shell-forming organisms to form $\mathrm{CaCO}_{3}$ shells, gets deposited on the ocean floor and makes its way back into the atmosphere via volcanic activity and plate tectonics. ${ }^{1}$, 7 The presence of other greenhouse gases is equally problematic. 8

\section{A Varying Gravitational Constant}

The seeming contradiction between a faint young Sun on the one hand, and support for a temperate climate on early Earth (and Mars) on the other, can easily be reconciled if one notes that the Sun's luminosity is exceedingly sensitive to the value of the gravitational constant. Indeed, as first pointed out by Teller, 10 the luminosity of the Sun, $\mathcal{L}_{\odot}$, depends upon its mass, $M_{\odot}$, and Newton's gravitational constant, $G$, as $\mathcal{L}_{\odot} \propto G^{7} M_{\odot}^{5}$. Increasing the value of $G$, one increases the solar luminosity. Moreover, a larger value of $G$ affects the Earth's orbit by bringing it closer to the Sun. The (conserved) specific angular momentum on a circular orbit of radius $r$ with velocity $v$ is $v r$. Since $v^{2} r^{2}=G M_{\odot} r$, it follows that the radius of the Earth's (almost circular) orbit $r \propto 1 / G M_{\odot}$. Since flux varies with distance as $\mathcal{F}_{\odot} \propto r^{-2}$, the amount of sunlight received on Earth becomes

$$
\mathcal{F}_{\odot} \propto G^{9} M_{\odot}^{7}
$$


which translates into $T \propto \mathcal{F}_{\odot}^{1 / 4} \propto G^{2.25} M_{\odot}^{1.75}$ for Earth's temperature ${ }^{10 \mid 12}$ (also see Ref. (13). From (11), one gets

$$
\frac{\Delta \mathcal{F}_{\odot}}{\mathcal{F}_{\odot}}=9 \frac{\Delta G}{G}+7 \frac{\Delta M_{\odot}}{M_{\odot}} .
$$

Substituting $\Delta \mathcal{F}_{\odot} / \mathcal{F}_{\odot}=0.25$, one finds that a modest increase of $2 \%$ in the value of the gravitational 'constant' over a lookback time of 4 Gyr would largely compensate for the diminished sunlight on Earth. This scenario, then, would allow for Earth's temperature to remain roughly constant upto the present, in agreement with current data and without invoking the need for injecting enormous amounts of greenhouse gases into Earth's early atmosphere, for which no compelling evidence exists. ${ }^{8}$

Our estimate of $\Delta G / G \simeq 0.02$ at a lookback time of $\sim 4$ Gyr is in excellent agreement with the current observational constraints on varying $G$, including the results of $\mathrm{Wu}$ and Chen,, 14 who obtain $-0.083<\Delta G / G<0.095$ at Recombination, and those of Accetta et al. $\frac{15}{15}$ who obtain $-0.3<\Delta G / G<0.4$ at Nucleosynthesis. Since the above estimate of $\Delta G / G$ is model independent, one cannot directly relate it to the current value of $\dot{G} / G$. Nevertheless, if one assumes, for simplicity, $G \propto$ $t^{-\beta}$, then one finds $\dot{G} /\left.G\right|_{t_{0}} \simeq-4 \times 10^{-12} \mathrm{yr}^{-1}$, which is in good agreement with binary pulsar data, $\frac{16}{16}$ millisecond pulsar data,, 17 ages of globular clusters $\frac{18}{18}$ and lunar ranging experiments $\frac{19}{19}$

\section{Theories with Varying $G$}

The idea that $G$ may be a function of time was first suggested by Dirac who postulated $G \propto t^{-1}$ as part of his Large Numbers Hypothesis. ${ }^{20}$ Although such a large variation in $G$ was shown to be inconsistent with observations, Dirac's hypothesis was adopted into a field-theoretic framework in which Newton's constant acquired the status of a dynamical field.21]22

The action for the gravity sector of such a scalar-tensor theory is 12

$$
S=\frac{1}{16 \pi G_{*}} \int\left[F(\varphi) R-Z(\varphi)(\partial \varphi)^{2}-2 U(\varphi)\right] \sqrt{-g} \mathrm{~d}^{4} x,
$$

where $G_{*}$ is the bare gravitational constant. Comparing this with the EinsteinHilbert action for general relativity,

$$
S=\frac{1}{16 \pi G} \int R \sqrt{-g} \mathrm{~d}^{4} x,
$$

one finds that the role of the gravitational constant is played by $G=G_{*} / F(\varphi)$, where the scalar $\varphi$ satisfies the equation of motion

$$
2 Z(\varphi) \square \varphi=-\frac{d F}{d \varphi} R-\frac{d Z}{d \varphi}(\partial \varphi)^{2}+2 \frac{d U}{d \varphi} .
$$

One therefore needs to solve (5) in order to determine the time-dependence of $G[\phi(t)]$. This has indeed been done for specific choices of $U$ and $F$, including $F=$ $\xi \varphi^{2}$ (induced gravity) and $F=1+\xi \varphi^{2}$ (non-minimal coupling) ${ }^{121}$ Interestingly, for 
a suitable choice of $U$, such a model can describe dark energy (DE) by making the universe accelerate at late times. 23

A varying gravitational constant also arises in higher-dimensional Kaluza-Klein theories. For instance, the 5D Einstein-Hilbert action (described by the 5D metric $\left.\bar{g}_{A B}\right)$

$$
S=\frac{1}{12 \pi^{2} G_{5}} \int \bar{R} \sqrt{-\bar{g}} \mathrm{~d}^{5} x
$$

when compactified onto $4 \mathrm{D}$ via the decomposition

$$
\bar{g}_{A B}=\left(\begin{array}{cc}
g_{\mu \nu}+\frac{A_{\mu} A_{\nu}}{M^{2}} \phi^{2} & \frac{A_{\mu}}{M} \phi^{2} \\
\frac{A_{\nu}}{M} \phi^{2} & \phi^{2}
\end{array}\right),
$$

results in the $4 \mathrm{D}$ action

$$
S=\frac{1}{16 \pi G_{*}} \int\left(R-\frac{\phi^{2}}{4 M^{2}} F^{2}\right) \phi \sqrt{-g} \mathrm{~d}^{4} x .
$$

Comparing (6) with (4), one finds that $G \propto \phi^{-1}$, which generalizes to $G \propto \phi^{-D}$ in the case of $D$ extra dimensions.

One should note that scalars such as $\phi$ are ubiquitous in string theory, where they occur in the form of a dilaton whose vacuum expectation value determines the coupling constants of string theory ${ }^{27}$ In such theories, which include $\mathrm{SO}(32)$ and $E_{8} \times E_{8}$ heterotic theories, one usually expects the $4 \mathrm{D}$ coupling constants, including $G$, to vary with time.

\section{Conclusions}

The faint young Sun hypothesis implies that during much of prehistory-from 4 Gyr to 1 Gyr ago - both Earth and Mars would have been frozen. While the presence of water on early Earth is well documented, the presence of outflow channels and phyllosillicates, discovered by the Mars Reconnaissance Orbiter and by the Mars Exploration Rovers, also point to abundant liquid water on early Mars ${ }^{1|28|}$ Consequently, the faint young Sun presents a paradox for both Earth and Mars.1]

We have shown that tiny changes in $G$ can resolve the faint young Sun paradox by increasing the Sun's luminosity and bringing the Earth closer to the Sun 4 Gyr ago.

A variable gravitational constant has other important consequences. The peak luminosity of type Ia supernovae (SNIa) is proportional to the Chandrasekhar mass $M_{\mathrm{Ch}}$, which depends upon Newton's constant. Consequently, $\mathcal{L} \propto M_{\mathrm{Ch}} \propto G^{-3 / 2}$, and a larger value of $G$ in the past would result in SNIa being fainter than predicted by the standard candle hypothesis. ${ }^{29 \mid 30}$ A varying $G$ would therefore imply a new source of systematics in SNIa data. This would show up as tension between data sets probing DE using standard rulers $(\mathrm{BAO}+\mathrm{CMB})$ on the one hand, and standard candles (SNIa) on the other, with the latter predicting a more phantom-like equation 
of state for DE. Precisely such a tension has recently been reported by the Planck team. 31

Models with variable $G$ may also resolve the cosmological constant problem $\frac{\sqrt{32}}{3}$ Self-tuning mechanisms can dynamically reduce the vacuum energy to a small value. In doing so, they invariably turn the gravitational constant into a variable quantity ${ }^{\sqrt[33]{35}}$ Thus, the faint young Sun paradox, the cosmological constant problem and dark energy may all be pieces of the same puzzle requiring a common theoretical platform for their resolution.

\section{Acknowledgments}

The authors acknowledge support from the India-Ukraine Bilateral Scientific Cooperation programme. We acknowledge useful discussions with Adrian Melott and Prasad Subramanian.

\section{References}

1. J. I. Lunine, Earth, Evolution of a Habitable World (Cambridge University Press, Cambridge, UK, 2013).

2. D. O. Gough, Solar Physics 74 (1) (1981) 21.

3. C. Sagan and G. Mullen, Science 177 (4043) (1972) 52.

4. L. P. Knauth, Origin and diagnosis of cherts: an isotopic perspective, in Isotopic Signtures and Sedimentary Records, eds. N. Clauer and S. Chaudhuri (Springer-Verlag, Berlin, 1992), pp. 123-152.

5. L. P. Knauth, Palaeogeography, Palaeoclimatology, Palaeoecology 219 (2005) 53.

6. P. Falkowski et al., Science 290 (2000) 291.

7. J. F. Kasting and T. P. Ackerman, Science 234 (1986) 1383.

8. M. T. Rosing et al., Nature 464 (2010) 744.

9. C. Goldblatt and K. J. Zahnle, Nature 474 (2011) E1.

10. E. Teller, Phys. Rev. 73 (1948) 801.

11. G. Gamow, Phys. Rev. Lett. 19 (1967) 759.

12. J.-P. Uzan, Living Rev. Relativity 14 (2011) 2.

13. L. Iorio, Galaxies 1(3) (2013) 192.

14. F. Wu and X. Chen, Phys. Rev. D 82 (2010) 083003.

15. F. S. Accetta, L. M. Krauss and P. Romanelli, Phys. Lett. B 248 (1990) 146.

16. V. M. Kaspi, J. H. Taylor and M. F. Riba, Astrophys. J. 428 (1994) 713.

17. J. P. W. Verbiest et al., Astrophys. J. 679 (2008) 675.

18. S. Degl'Innocenti et al., Astron. Astrophys. 312 (1996) 345.

19. J. G. Williams, X. X. Newhall and J. O. Dickey, Phys. Rev. D 53 (1996) 6730.

20. P. A. M. Dirac, Nature (London) 139 (1937) 323.

21. P. Jordan, Naturwissenschaften 25 (1937) 513.

22. C. Brans, and R. H. Dicke, Phys. Rev. 124 (1961) 925.

23. L. Amendola and S. Tsujikawa, Dark Energy (Cambridge University Press, Cambridge, 2010).

24. V. Sahni and S. Habib, Phys. Rev. Lett. 81 (1988) 1766.

25. F. Perrotta, C. Baccigalupi and S. Matarrese, Phys. Rev. D 61 (1999) 023507.

26. S. Tsujikawa et al., Phys. Rev. D 77 (2008) 103009.

27. E. Witten, Phys. Lett. B 149 (1984) 351. 
28. C. P. McKay and W. L. Davis, Icarus 90 (1991) 214.

29. E. Gaztañaga et al., Phys. Rev. D 65 (2001) 023506.

30. A. Riazuelo and J.-P. Uzan, Phys. Rev. D 66 (2002) 023525.

31. P. A.R. Ade et al. [Planck Collaboration], Planck 2013 results. XVI. Cosmological parameters, arXiv:1303.5076 [astro-ph.CO].

32. S. Weinberg, Rev. Mod. Phys. 61 (1989) 1.

33. A. D. Dolgov, An attempt to get rid of the cosmological constant, in The Very Early Universe, eds. G. W. Gibbons, S. W. Hawking and S. T. C. Siklos (Cambridge University Press, Cambridge, 1983), pp. 449-458.

34. C. Charmousis et al., Phys. Rev. Lett. 108 (2012) 051101.

35. J. Sola, Vacuum energy and cosmological evolution, arXiv:1402.7049 [gr-qc]. 